\section{The Correlation of Visibilty Noise and Its Impact on the Radiometric Resolution of an Aperture Synthesis Radiometer}

Javier Bará, Adriano Camps, Francesc Torres, and Ignasi Corbella

\begin{abstract}
The correlation between the visibility samples' noise of an aperture synthesis radiometer are required for the computation of the recovered temperature noise of a given pixel and of the improvement introduced by baseline redundance. A general expression for this correlation and noise examples for a linear array are presented.
\end{abstract}

Index Terms-Microwave radiometry, microwave receivers, radio interferometry, radiometry, remote sensing, synthetic aperture imaging.

\section{INTRODUCTION}

The interest in the global radiometric measurement of ocean salinity and soil moisture in the lower microwave range (L-band), and the preference of two-dimensional (2-D) aperture synthesis over total power radiometry have been pointed out recently [1]. The progress of the NASA since 1987 with their prototype ESTAR and, more recently, of the European Space Agency (ESA) with the design of the 2-D aperture synthesis radiometer microwave imaging radiometer by aperture synthesis (MIRAS) are also well documented. It has actually been this latter project that prompted an investigation of the achivable radiometric sensitivity of a complex instrument with 133 antennas and more than 10000 correlators.

The output of a real aperture radiometer is proportional to its antenna temperature plus the receiver equivalent noise temperature (system temperature). The radiometer nonidealities (of which finite integration time is unavoidable) produce measurement uncertainties that are frequently quantified through the concepts ofradiometric sensitivity, radiometric resolution, or SNR [2]-[4]. To be specific, the radiometric resolution (or sensitivity) is defined as the smallest change in the average brightness temperature that can be detected by the instrument [4]. Following generally accepted criteria, this smallest change is taken as the standard deviation of the random perturbations at the instrument output. In this way it is found that, after the exploration of a scene by the real aperture radiometer, the radiometric sensitivity of a given pixel is proportional to its system temperature.

In the case of an aperture synthesis radiometer, the expressions commonly found in the literature for its radiometric resolution provide, in all cases of interest, the same value for all the pixels in the synthetic image, a fact that is the consequence of the approximate assumption of uncorrelation of visibility noise. The purpose of this work is to obtain a better approximation for the radiometric sensitivity of the pixels of a synthetic image when the correlation between visibility noise is considered. This correaltion also affects the radiometric sensitivity improvement introduced by redundant baselines.

\section{BASIC BASELINE EQUATION}

In an ideal aperture synthesis radiometer formed by identical antennas and receivers with a very narrow bandwidth, and with an infinite

Manuscript received March 1, 1996; revised March 4, 1999.

J. Bará, A. Camps, F. Torres, and I. Corbella are with the Department of Signal Theory and Communications, Polytechnic University of Catalonia, Barcelona, Spain (e-mail: bara@tsc.upc.es).

Publisher Item Identifier S 0196-2892(00)06230-6. integration time, the visibility function $V(u, v)$ is the Fourier transform of the "modified brightness temperature," $T(\xi, \eta)$ through [5]

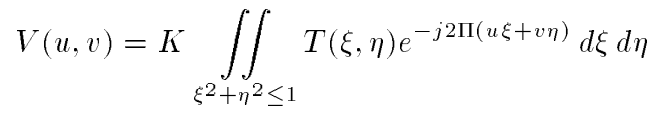

with

$$
T(\xi, \eta)=\frac{T_{B}(\xi, \eta)}{\sqrt{1-\xi^{2}-\eta^{2}}}\left|F_{n}(\xi, \eta)\right|^{2}
$$

and where $(u, v)$ are the spatial frequencies, $T_{B}$ is the brightness temperature, $(\xi, \eta)$ are the director cosines of a given direction, $F_{n}$ is the antenna normalized voltage pattern, and $K$ is a constant depending on the receivers and correlators gain.

It has been shown elsewhere that the effects of finite bandwith (fringe-washing) can be taken into account by replacing the actual modified brightness temperature by a blurred version $\bar{T}(\xi, \eta)$ [6]. Therefore, without loss of generality, we will use (1) as the basic equation relating the image to the visibility function.

When we do not have the full information of $V(u, v)$ but rather $M=$ $N_{u} N_{v}$ visibility samples $V_{m n}$ equispaced over a parallelogram, we compute the following estimation $\hat{T}$ of the modified temperature:

$$
\begin{aligned}
K \hat{T}\left(\xi_{i}, \eta_{k}\right) \doteq & K \hat{T}_{i k}=\sqrt{M} \Delta s \\
& \times \sum_{m=1}^{N_{u}} \sum_{n=1}^{N_{v}} F_{\left(N_{u}\right) i m} F_{\left(N_{v}\right) k n} W_{m n} V_{m n}
\end{aligned}
$$

where $\Delta s$ is the elementary area in the $(u, v)$ plane, $W_{m n}$ is the chosen weighting function, and $F_{(N)}$ is the $N$-th order symmetric discrete Fourier transform (DFT) matrix given by

$$
F_{(N) i m}=N^{-1 / 2} e_{N}^{(i-1)(m-1)}, \quad e_{N}=e^{j 2 \pi / N} .
$$

Note that this expression is valid for both a rectangular grid and a hexagonal grid with a proper definition of the lattices in the $(u, v)$ and $(\xi, \eta)$ spaces [7]. For the sake of simplicity, and without loss of generality, we will assume that $N_{u}=N_{v}=N$ and will omit it in the summations when it is clearly understood.

In the presence of additive noise $n_{i}(t)$ in the transmission channels, the visibility values, now denoted by a superindex $n$, do not change, except the baseline $V(0,0)=V_{11}$ (refer to paragraph IV)

$$
\begin{aligned}
& V^{n}(u, v)=\left\langle\left(s_{i}+n_{i}\right)\left(s_{j}^{*}+n_{j}^{*}\right)\right\rangle=V(u, v) \\
& V^{n}(0,0)=V_{11}^{n}=\left\langle\left|s_{i}\right|^{2}\right\rangle+\left\langle\left|n_{i}\right|^{2}\right\rangle=V_{11}+\left\langle|n|^{2}\right\rangle .
\end{aligned}
$$

\section{IMAGE SENSITIVITY AND REDUNDANCY CONCEPTS}

When the visibility samples have errors $\Delta V_{m n}$, these translate into estimated temperature errors given by the matrix equation

$$
\begin{aligned}
K \Delta \hat{\mathbf{T}} \doteq K \Delta \mathbf{T}=\sqrt{M} \Delta s \mathbf{F} \Delta \mathbf{V}_{\boldsymbol{W}} \mathbf{F}^{\mathbf{t}}, \\
{\left[\mathbf{V}_{\boldsymbol{W}}\right]_{m n}=\Delta V_{m n} W_{m n} . }
\end{aligned}
$$

Let's define the scene's average modified brightness temperature error $(\Delta T)_{\text {avg by }}$

$$
\begin{aligned}
(\Delta T)_{\mathrm{avg}}^{2} & =\frac{1}{M} \sum_{m} \sum_{n}\left\langle\left|\Delta T_{m n}\right|^{2}\right\rangle \\
& =\left(\frac{\Delta s}{K}\right)^{2} \sum_{m} \sum_{n} W_{m n}^{2}\left\langle\left|\Delta V_{m n}\right|^{2}\right\rangle .
\end{aligned}
$$


In this transformation, we have taken into account that a DFT preserves the Euclidean norm. Let us see how this definition leads to the known signal to noise expressions. When considering the errors produced by the finite integration time $\tau$ (defined as in (26)), we speak of noise and we have [[8], (12) and (13)]:

$$
\begin{aligned}
\left\langle\left|\Delta V_{m n}\right|^{2}\right\rangle & =\frac{\left(V_{11}^{n}\right)^{2}}{B \tau} \rightarrow(\Delta T)_{\mathrm{avg}} \\
& =\frac{V_{11}^{n} \Delta s}{K} \sqrt{\frac{M}{B \tau}} \beta_{W}
\end{aligned}
$$

where

$$
\begin{aligned}
V_{11}^{n} & =K T_{\mathrm{sys}} \Omega_{p}=K T_{\mathrm{sys}} \frac{\lambda^{2}}{A_{e}}, \\
\beta_{W} & =\sqrt{\frac{1}{M} \sum_{m} \sum_{n} W_{m n}^{2}}
\end{aligned}
$$

with $B$ the receiver bandwidth [[9], (7c)], $\Omega_{p}$ the pattern equivalent solid angle of each of the receiving antennas, $A_{e}$ its effective area, and $T_{\text {sys }}$ the system equivalent temperature [5]. Therefore

$$
\begin{aligned}
\frac{(\Delta T)_{\mathrm{avg}}}{T_{\mathrm{sys}}} & =\Omega_{p} \Delta s \beta_{W} \sqrt{\frac{M}{B \tau}} \\
& =\frac{A_{\mathrm{syn}}}{A_{e}} \frac{\beta_{W}}{\sqrt{M B \tau}} . \quad\left(A_{\mathrm{syn}}=M \Delta s \lambda^{2}\right)
\end{aligned}
$$

When no windows are used, $\beta_{W}=1$, and one of the forms for this equation found in the literature is recovered [10].

If we are interested in the rms noise of a given pixel we must compute

$$
\begin{aligned}
K^{2}\left\langle\left|\Delta T_{m n}\right|^{2}\right\rangle= & (\Delta s)^{2} \sum_{i} \sum_{k} \sum_{r} \sum_{s} W_{i k} W_{r s} \\
& \times\left\langle\Delta V_{i k} \Delta V_{r s}^{*}\right\rangle e_{N}^{(m-1)(i-r)+(n-1)(k-s)}
\end{aligned}
$$

and a knowledge of the correlation between noise signals is required. If these are assumed uncorrelated, the value given by (7)-(10) is recovered. That is, the approximation of noise uncorrelation gives for every pixel a modified temperature noise equal to the scene average noise.

If a visibility point $V_{m n}$ is measured by $P$ baselines (that is, if is $P$-fold redundant), each yielding a value $V_{m n}^{(\alpha)}$, we can take its average and then

$$
\begin{aligned}
\overline{\Delta V_{m n}} & =\frac{1}{P} \sum_{\alpha=1}^{P} \Delta V_{m n}^{(\alpha)}, \\
\left\langle\left|\overline{\Delta V_{m n}}\right|^{2}\right\rangle & =\frac{1}{P^{2}} \sum_{\alpha} \sum_{\beta}\left\langle\Delta V_{m n}^{(\alpha)} \Delta V_{m n}^{(\beta) *}\right\rangle
\end{aligned}
$$

and the correlation between the visibility noise is again required. Previous works at this point assume that the noise signals of different baselines are uncorrelated, and then the visibility error variance is reduced by the number of baselines $P$ [8].

\section{Correlation Between Noise Signals}

It turns out that the visibility noise signals can be strongly correlated, and its computation is this paper's main result. Let us consider four antennas labeled 1, 2, 3, and 4. Antennas 1 and 2 produce the following estimation visibility sample by integration in the time interval $(t, t+\tau)$ :

$$
\begin{aligned}
\hat{V}_{(1,2)}= & \int_{-\infty}^{\infty} a(t-p)\left[s_{1}(\rho)+n_{1}(\rho)\right] \\
& \times\left[s_{2}^{*}(\rho)+n_{2}^{*}(\rho)\right] d \rho
\end{aligned}
$$

where the subindeces within parentheses $(1,2)$ refer to the antennas forming the baseline, $s_{i}$ is the signal from the channel in the absence of noise, $n_{i}$ is the noise introduced by the amplifying/downconverting chain, and $a(t)$ is the postmultiplication, low-pass filter impulse response, which fixes the integration time $\tau$. A similar expression holds for the baseline $(3,4)$. It is shown in the Appendix that

$$
\left\langle\Delta V_{(1,2)} \Delta V_{(3,4)}^{*}\right\rangle=\frac{V_{(1,3)} V_{(2,4)}^{*}}{B \tau} .
$$

This expression is still valid if the two baselines share an antenna, or even both of them [in this latter case, we recover the expression for the baseline noise (8)], if whenever in the expression it appears $V(0,0)=V_{11}$, its value is replaced by $V_{11}^{n}$ as given by (5). Note that this correlation is a complex number and that, for a scene formed by a single delta function, its absolute normalized value is always unity (full correlation).

\section{ONE-DimENSIONAL (1-D) EXAMPLES}

Inclusion of noise correlation in the computation of both radiometric resolution and redundancy impact requires a knowledge of the spatial position of the antennas forming each baseline, that is, a knowledge of the specific radiometer configuration and baseline forming strategy, which makes it impossible to proceed with a general analysis. To reduce the analytic complexity in the examination of examples, we turn our attention to a one-dimensional (1-D) or linear array, for which (1) becomes

$$
V(u)=K \int_{-1}^{1} \frac{T_{B}(\xi)}{\sqrt{1-\xi^{2}}}\left|F_{n}(\xi)\right|^{2} e^{-j 2 \pi u \xi} d \xi .
$$

Complexity is kept at its lowest if for the antenna normalized voltage pattern we assume

$$
\begin{aligned}
\left|F_{n}(\xi)\right|^{2} & =\sqrt{1-\xi^{2}}=\cos \theta \rightarrow \Omega_{p} \\
& =\int_{-1}^{1} \frac{\left|F_{n}(\xi)\right|^{2}}{\sqrt{1-\xi^{2}}} d \xi=2=\frac{\lambda}{L_{e}}
\end{aligned}
$$

where $L_{e}$ is the antenna effective length. In this way

$$
\begin{aligned}
V(u) & =K \int_{-1}^{1} T_{B}(\xi) e^{-j 2 \pi u \xi} d \xi, \quad V(0)=2 K T_{B \text { avg' }} \\
V^{n}(0) & =2 K\left(T_{B \text { avg }}+T_{n}\right) .
\end{aligned}
$$

That is, what we have called modified temperature matches in this case the brightness temperature.

We shall consider a linear array formed by $N$ equispaced antennas numbered from 1 to $N$. The measured visibility samples are $V_{m}=$ $V[(m-1) \Delta u], m=1,2, \ldots N$, but since $V(-n \Delta u)=V^{*}(n \Delta u)$, the negative index visibilities are also available. To avoid the problems brought into by aliasing we will assume that the antennas are spaced by a half wavelength, that is, $\Delta u=1 / 2$.

\section{A. Example 1-Global versus Pixel Sensitivity in Nonredundant Array}

Let us first assume that $V_{m}$ is measured only once (zero redundancy array) with antennas $(1, m)$. That is

$$
V_{m}=V[(m-1) \Delta u]=V_{(1, m)^{\prime}} \quad 1 \leq m \leq N .
$$

To examine the behavior of pixel noise as predicted by (11), we constructed the brightness temperature distribution of Fig. 1, left, which shows a sharp transition from $90 \mathrm{~K}$ (representative value for the sea) to $250 \mathrm{~K}$ (idem for land) and another jump from 250 to $200 \mathrm{~K}$. We chose $N=65=2^{6}+1$, which for a frequency of $1.4 \mathrm{Ghz}$ gives an array 

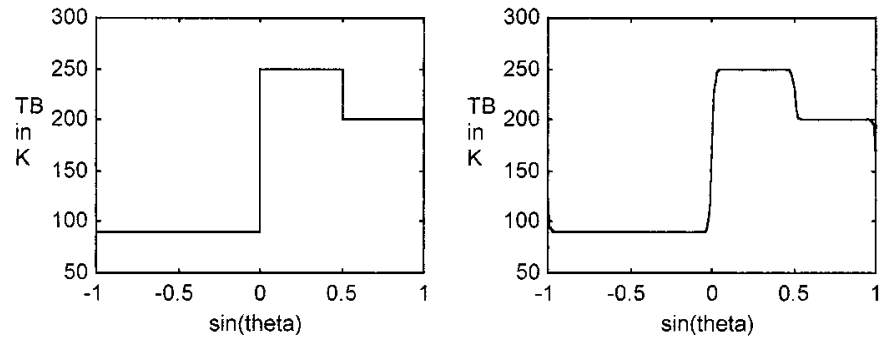

Fig. 1. (Left) One-dimensional (1-D) brightness temperature distribution used in the examples. $\theta$ is the angle as measured from boresight. (Right) Recovered distribution with 65 antennas and a Blackmann window.

length of $6.86 \mathrm{~m}$, somewhat smaller than one of the MIRAS project's arms $(8.2 \mathrm{~m})$. The recovered temperature distribution for a Blackmann window is also shown in Fig. 1, right. Fig. 2 shows the results obtained from (11) for the brightness temperature error normalized to $B \tau=1$, and for a receiver noise equivalent temperature of $T_{n}=50 \mathrm{~K}(0.7 \mathrm{~dB}$ noise figure, corresponding to a good quality low noise L-band amplifier).

In order to assess the results obtained, we define an equivalent real aperture scanning radiometer (ERASR) as that with identical bandwidth, spatial resolution, and integration time for a given scene as our synthesized radiometer. Therefore, its integration time for a given observation (single pixel) will be

$$
\tau^{\prime}=\frac{\tau}{M \beta_{W}^{2}} . \quad M=2 N-1
$$

Note that the window factor appears since it affects the spatial resolution (uncertainty principle [9]). It can be seen that the temperature errors of the synthesized radiometer follow the shape of the temperature distribution, but, as compared to the ERASR, they are higher in the colder regions (sea) and lower in the warmer (land), Fig. 2.

\section{B. Example 2-Fully Redundant Array}

By this, we will understand a linear array, as in example 1, where all possible baselines between the available antennas are measured. That is, the baseline $V_{m}=V_{(k, k+m)}$ is measured for $k=1,2,3, \ldots N-m$, and the sample redundancy is $N-m$. Therefore, we define the visibility sample average value by

$$
\begin{aligned}
\bar{V}_{m}=\bar{V}_{-m}^{*}=\frac{1}{N-m} \sum_{k=1}^{N-m} V_{(k, k+m)^{\prime}} \\
\quad m=1,2,3, \ldots N .
\end{aligned}
$$

For the same linear array as in the example 1, (11) provides normalized brigtness temperature errors shown in Fig. 2, right. It can be seen how these errors again follow the shape of the temperature distribution but with a substantially lower average value compared to the nonredundant array, at the expense of increasing the number of correlators in the system from $2 \times 65=130$ to $2 \times 2080=4160$ (that is, by a factor of 32). Fig. 2 shows also the errors under the assumption of error uncorrelation, which coincide with the scene average error as given by (7).

\section{CONCLUSION}

It has been shown how the correlation between the noise signals of the visibility samples taken in the same time interval in an aperture synthesis radiometer both modifies the resolution improvement obtained through baseline redundancy and plays an important role in the computation of the radiometric resolution of every pixel for a given brightness distribution scene. An explicit expression for this correlation has
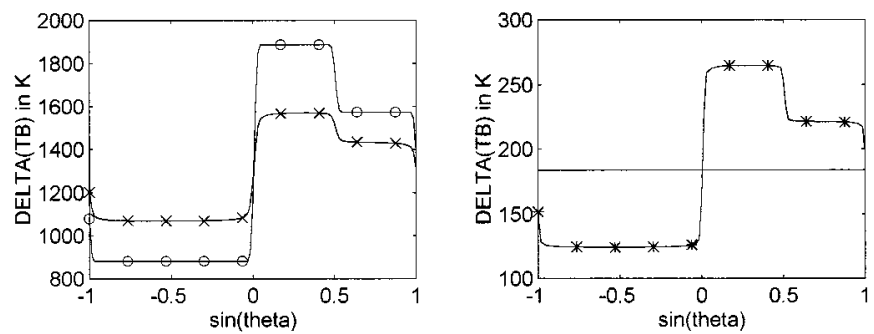

Fig. 2. Normalized values $(B \tau=1)$ of the pixel temperature errors obtained for the examples. (x): Non-redundant array. (o): Equivalent real aperture scanning radiometer (ERASR). (*): Fully redundant array. (-): Fully redundant array, visibility error correlation neglected.

been given which, for a specific radiometer configuration and baseline geometrical organization, allows the computation of the pixels' random errors (noise). A particular, mathematically simple 1-D nonredundant aperture synthesis radiometer (the baselines are formed by one of the outermost antennas and all the rest) has been studied. In this case, the analysis shows that the noise errors follow the brightness temperature profile and are therefore below the average value in the colder pixels and above it in the warmer ones, although the variations are smaller than those of an equivalent real aperture scanning radiometer (ERASR). The analysis was extended to the same linear array but with full redundance, and this shows again how noise tends to follow the temperature profile.

These analyses are examples of how maps of temperature noise, rather than a single figure for its average value, can be produced for a synthetic aperture radiometer.

\section{APPENDIX}

Let us first recall that, for an arbitrarily narrowband system (negligible spatial decorrelation) [7]

$$
\begin{aligned}
V_{(i, j)}(\chi)= & \left\langle s_{i}(t+\chi) s_{j}^{*}(t)\right\rangle \\
= & K \iiint_{\xi^{2}+\eta^{2} \leq 1} T(\xi, \eta) \tilde{r}\left(\chi-\frac{u \xi+v \eta}{f_{o}}\right) \\
& \times e^{-j 2 \pi(u \xi+v \eta)} d \xi d \eta \\
\approx & \tilde{r}(\chi) V_{(i, j)}(0)
\end{aligned}
$$

where \langle\rangle denotes ensemble average. Note that the actual visibility samples are these variables evaluated for $\chi=0$. Now, following (13), let us compute the correlation of two visibility estimations

$$
\begin{aligned}
& \left\langle\hat{V}_{(1,2)}(t) \hat{V}_{(3,4)}^{*}(t)\right\rangle \\
& =\iint a(t-\rho) a(t-\sigma)\left\langle\left[s_{1}(\sigma)+n_{1}(\sigma)\right]\right. \\
& \quad \times\left[s_{2}^{*}(\sigma)+n_{2}^{*}(\sigma)\right]\left[\left(s_{3}^{*}(\rho)+n_{3}^{*}(\rho)\right]\right. \\
& \left.\quad \times\left[s_{4}(\rho)+n_{4}(\rho)\right]\right\rangle d \rho d \sigma
\end{aligned}
$$

where both the $s_{i}$ and the $n_{i}$ are complex, zero mean Gaussian random processes with circular joint Gaussian statistics, for which ([11])

$$
\left\langle a_{1} a_{2} a_{3}^{*} a_{4}^{*}\right\rangle=\left\langle a_{1} a_{3}^{*}\right\rangle\left\langle a_{2} a_{4}^{*}\right\rangle+\left\langle a_{1} a_{4}^{*}\right\rangle\left\langle a_{2} a_{3}^{*}\right\rangle
$$

In the integrand of (22), there are 16 products, of which, 15 have at least one of the $n_{i}$. Since each $n_{i}$ is uncorrelated with all the remaining processes, when taking expected values, these 15 terms vanish and we 
are left only with

$$
\begin{aligned}
\left\langle s_{1}(\sigma) s_{2}^{*}(\sigma) s_{3}^{*}(\rho) s_{4}(\rho)\right\rangle \\
=\left\langle s_{1}(\sigma) s_{2}^{*}(\sigma)\right\rangle\left\langle s_{3}^{*}(\rho) s_{4}(\rho)\right\rangle \\
\quad+\left\langle s_{1}(\sigma) s_{3}^{*}(\rho)\right\rangle\left\langle s_{2}^{*}(\sigma) s_{4}(\rho)\right\rangle \\
=V_{(1,2)} V_{(3,4)}^{*}+V_{(1,3)} V_{(2,4)}^{*}|\tilde{r}(\sigma-\rho)|^{2} .
\end{aligned}
$$

Therefore, (22) becomes

$$
\begin{aligned}
& \left\langle\hat{V}_{(1,2)}(t) \hat{V}_{(3,4)}^{*}(t)\right\rangle \\
& =\iint a(t-\rho) a(t-\sigma)\left[V_{(1,2)} V_{(3,4)}^{*}\right. \\
& \left.\quad+V_{(1,3)} V_{(2,4)}^{*}|\tilde{r}(\sigma-\rho)|^{2}\right] d \sigma d \rho .
\end{aligned}
$$

Note that the low-pass filter may have an integration time of the order of $0.1 \mathrm{~s}$, while if $B \approx 20 \mathrm{MHz}, 1 B_{-1} \approx 5 \times 10^{-8} \mathrm{~s}$. Therefore, in (25) $a(t-\rho) a(t-\sigma)$ varies very slowly as compared to $|\tilde{r}(\sigma-\rho)|^{2}$, and we can approximate this latter by $1 B_{-1}$ times a delta function. Then

$$
\begin{aligned}
& \int_{-\infty}^{\infty} a(t-\sigma) d \sigma \int_{-\infty}^{\infty} a(t-\rho)|\tilde{r}(\rho-\sigma)|^{2} d \rho \\
& \quad \approx \frac{1}{B} \int_{-\infty}^{\infty} a^{2}(t-\sigma) d \sigma \\
& \quad=\frac{1}{B} \int_{-\infty}^{\infty}|A(f)|^{2} d f=\frac{1}{B \tau}
\end{aligned}
$$

$[A(f)$ being the Fourier transform of $a(t)]$, and (25) can be written

$$
\left\langle\hat{V}_{(1,2)} \hat{V}_{(3,4)}\right\rangle=V_{(1,2)} V_{(3,4)}^{*}+\frac{V_{(1,3)} V_{(2,4)}^{*}}{B \tau}
$$

from which (14) follows inmediately.

If the spatial decorelation effects are not negligible, it has been mentioned above that its effects can be taken into account by replacing the modified temperature $T(\xi, \eta)$ by a blurred version [6]. Therefore, we can expect (14) to continue being valid if the visibilities are computed from this blurred version.

\section{REFERENCES}

[1] M. Martin-Neira and E. Attema, "Conclusions and recommendations," presented at the Soil Moisture and Ocean Salinity Measurements and Radiometer Techniques Consultative Meeting, Noordwijk, The Netherlands, Apr. 20-22, 1995.

[2] D. M. LeVine, "The sensitivity of synthetic aperture radiometers for remote sensing from space," Radio Sci., vol. 25, no. 4, pp. 441-453, July-Aug. 1990.

[3] C. T. Swift, "ESTAR: The Electronically Scanned Thinned Array Radiometer for Remote Sensing Measurement of Soil Mositure and Ocean Salinity," NASA Tech. Memo. 4523, Washington, DC, 1993.

[4] F. T. Ulaby, R. K. Moore, and A. K. Fung, Microwave Remote Sensing. Norwood, MA: Artech House, 1981, vol. 1.

[5] F. Torres, A. Camps, J. Bará, I. Corbella, and R. Ferrero, "On-board phase and modulus calibration of large aperture synthesis radiometers: Study applied to MIRAS," IEEE Trans. Geosci. Remote Sensing, vol. 34, pp. 1000-1009, July 1996.

[6] J. Bará, A. Camps, F. Torres, and I. Corbella, "Angular resolution of twodimensional, hexagonally sampled interferometric radiometers," Radio Sci., vol. 33, pp. 1459-1473, Sept.-Oct. 1998.

[7] A. Camps, J. Bará, I. Corbella, and F. Torres, "The processing of hexagonally sampled signals with standard rectangular techniques: Application to 2-D large aperture synthesis interferometric radiometers," IEEE Trans. Geosci. Remote Sensing, vol. 35, pp. 183-190, Jan. 1997.

[8] C. S. Ruf, C. T. Swift, A. B. Tanner, and D. M. LeVine, "Interferometric synthetic aperture radiometry for the remote sensing of the earth," IEEE Trans. Geosci. Remote Sensing, vol. 26, pp. 597-611, Sept. 1988.
[9] A. Camps, I. Corbella, J. Bará, and F. Torres, "Radiometric sensitivity computation in aperture synthesis interferometric radiometry," IEEE Trans. Geosci. Remote Sensing, vol. 35, pp. 680-685, Mar. 1998.

[10] D. Le Vine, M. Kao, A. Tanner, C. Swift, and A. Griffis, "Initial results in the development of a synthetic aperture microwave radiometer," IEEE Trans. Geosci. Remote Sensing, vol. 28, pp. 614-619, July 1990.

[11] J. W. Goodman, Statistical Optics. New York: Wiley, 1985.

\section{Spatial Resolution Improvement of Remotely Sensed Images by a Fully Interconnected Neural Network Approach}

\author{
Maria del Carmen Valdes and Minoru Inamura
}

\begin{abstract}
In previous works, backpropagation neural networks (BPNN) had been applied successfully in the spatial resolution improvement of remotely sensed, low-resolution images using data fusion techniques [1]-[3]. However, the time required in the learning stage is long. In the present paper, a fully interconnected neural network (NN) model, valid from the mathematical and neurobiological points of view, is developed. With this model, the global minimum error is reached considerably faster than with any other method without regarding the initial settings of the network parameters.
\end{abstract}

Index Terms-Neural networks, spatial resolution improvement, thermal image.

\section{INTRODUCTION}

In contrast to conventional techniques, which are often limited by assumptions of normality, linearity, variable independence, etc., the advantages of neural networks $(\mathrm{NN})$ arise from their powerful pattern matching and nonlinear mapping capabilities with adaptive learning, tolerance to component failure, and robustness to noises.

In previous works [1]-[3], we demonstrated the usefulness of the backpropagation NN model in the spatial resolution improvement of remotely sensed images over the mathematical and statistical methods used up to the date with the same purposes.

However, a number of difficulties are associated with their use, restricting their general acceptability. Among them, we can mention the following.

1) The time required for minimizing the error in the learning process is too long.

2) These algorithms are not guaranteed to find the solution giving the global minimum, and the search procedure is unpredictable and may fall into local minima.

3) The level of accuracy that can be attained is usually totally unpredictable at the start of a training sequence and can also vary significantly depending on starting conditions for the training (i.e., network architecture or configuration, initial random weights settings, and training algorithm control parameter settings).

Manuscript received March 4, 1999; revised February 3, 2000. This research was supported in part by the Sasagawa Scientific Research Grant of The Japan Science Society, Tokyo.

The authors are with the Faculty of Engineering, Electronic Department, Gunma University, Gunma, Japan (e-mail: inamura@el.gunma-u.ac.jp).

Publisher Item Identifier S 0196-2892(00)06227-6. 